\title{
Drug-induced Hepatitis with Autoimmune Features during Minocycline Therapy
}

\author{
Masanori Abe, Shinya Furukawa, Shuzo Takayama, Kojiro MichitaKA*, Hisaka Minami, \\ Kazuhisa YAMAMOTO**, Norio HORIIKE and Morikazu ONJI
}

\begin{abstract}
A 25-year-old woman with no history of liver disease developed liver dysfunction associated with severe jaundice and general malaise following a prolonged therapy with minocycline for acne vulgaris. Serum anti-nuclear antibody was detected and immunoglobulin $G$ level was elevated. Symptoms resolved and liver function normalized following minocycline discontinuation and corticosteroid administration. Our diagnosis was drug-induced hepatitis with autoimmune features, as liver histology revealed acute hepatitis. Drug-induced hepatitis should be considered when liver dysfunction or systemic symptoms develops during long-term minocycline therapy.

(Internal Medicine 42: 48-52, 2003)
\end{abstract}

Key words: minocycline, drug-induced liver injury, longterm therapy, autoimmune hepatitis

\section{Introduction}

Minocycline hydrochloride is a semisynthetic tetracycline derivative used in the treatment of infectious disorders. It is particularly effective against Propionibacterium acnes and coagulase-negative staphylococci; thus, one of its most common indications is for the treatment of acne vulgaris. Under this indication (1), the drug is given for a prolonged period (months to years), mainly in young and otherwise healthy patients. The safety of minocycline in the treatment of acne has been widely reported $(2,3)$.

On the other hand, a variety of adverse effects related to minocycline treatment have been described. In recent years, it has become clear that minocycline can induce abnormal immune reactions, resulting in hepatitis, arthritis, and sys- temic lupus erythematous-like syndrome (4-13).

Some cases of autoimmune hepatitis (AIH) associated with minocycline have been reported in Europe and the United States (4-6, 8-13). With increasing recognition, the number of reported cases has expanded in the past several years. In Japan, however, there have been few reports of minocycline-induced AIH. We report here a 25-year-old patient with acute hepatitis with autoimmune features who had been taking minocycline for 12 months prior to the onset of disease.

\section{Case Report}

A 25-year-old Japanese woman was referred to our hospital on January 30, 2002 for investigation of deranged liver function tests associated with severe jaundice and general malaise. She had been prescribed minocycline for acne vulgaris from a local clinic and had been taking the drug, $100 \mathrm{mg}$ daily, for 1 year. During this period, she had never complained of any specific symptoms, and her liver function had never been tested. In December 2001, she developed general malaise. She had no history of drug or food allergies, blood transfusions or significant alcohol consumption.

On admission, physical examination was significant only for obvious icterus. She was alert and had no hepatosplenomegaly or other characteristics of chronic liver disease. Acne was present, however, no other skin rash was evident.

The results of her laboratory tests are shown in Table 1. The total white blood cell count was within the normal limit, without eosinophilia. Prothrombin time (PT) was prolonged. The level of total bilirubin was elevated to $23.7 \mathrm{mg} / \mathrm{dl}$. Serum aspartate aminotransferase (AST) and alanine aminotransferase (ALT) levels were elevated. The serum was negative for IgM-type antibodies against hepatitis A virus (HAV) and hepatitis B virus (HBV). Hepatitis C virus (HCV) RNA, hepatitis G virus (HGV) RNA, and TT virus (TTV) DNA

From the Third Department of Internal Medicine, *the Endoscopy Center, Ehime University School of Medicine, Ehime and **the Department of Gastroenterology, Matsuyama-Shimin Hospital, Matsuyama

Received for publication August 22, 2002; Accepted publication November 8, 2002

Reprint requests should be addressed to Dr. Morikazu Onji, the Third Department of Internal Medicine, Ehime University School of Medicine, Shigenobucho, Ehime 791-0295 
Minocycline-induced Hepatitis

Table 1. Laboratory Data on Admission

\begin{tabular}{clll}
\hline CBC & & Serological test & \\
WBC & $7,800 / \mu 1$ & anti-HA IgM & $(-)$ \\
Neutro & $65 \%$ & HBsAg & $(-)$ \\
Lymph & $21 \%$ & anti-HBc IgM & $(-)$ \\
Mono & $12 \%$ & anti-HBc $(\times 1)$ & $(+) 89$ \\
Eos & $1.5 \%$ & \multicolumn{1}{c}{$(\times 200)$} & $(-)$ \\
Baso & $0.5 \%$ & anti-HCV & $(-)$ \\
RBC & $437 \times 10^{4} / \mu 1$ & HCV-RNA & $(-)$ \\
Hgb & $14.3 \mathrm{~g} / \mathrm{dl}$ & HGV-RNA & $(-)$ \\
Hct & $43.0 \%$ & TTV-DNA & $(-)$ \\
Plate & $40.9 \times 10^{4} / \mu 1$ & ANA & $\times 160($ Diffuse $)$ \\
ESR & $18 \mathrm{~mm} / \mathrm{h}$ & ASMA & $<\times 20$ \\
& & AMA & $<\times 10$ \\
Coagulation tests & & p-ANCA & $<3.1$ \\
PT & $41.4 \%$ & anti-ssDNA & $<5$ \\
APTT & $38.0 \mathrm{sec}$ & anti-dsDNA & $<5$ \\
& & IgG & $2,110 \mathrm{mg} / \mathrm{dl}$ \\
Blood chemistry & & IgA & $196 \mathrm{mg} / \mathrm{dl}$ \\
T.prot & $6.8 \mathrm{~g} / \mathrm{dl}$ & IgM & $131 \mathrm{mg} / \mathrm{dl}$ \\
Alb & $3.8 \mathrm{~g} / \mathrm{dl}$ & anti-IgM-EA-EBV & $<\times 10$ \\
T.Bil & $23.7 \mathrm{mg} / \mathrm{dl}$ & anti-IgM-VCA-EBV & $<\times 10$ \\
D.Bil & $15.5 \mathrm{mg} / \mathrm{dl}$ & anti-IgM-CMV & $<\times 10$ \\
AST & $1,870 \mathrm{IU} / l$ & anti-IgM-HSV & $<\times 10$ \\
ALT & $2,074 \mathrm{IU} / l$ & anti-IgM-HHV-6 & $<\times 10$ \\
LDH & $562 \mathrm{IU} / l$ & & \\
ALP & $138 \mathrm{IU} / l$ & HLA studies & \\
$\gamma$-GTP & $28 \mathrm{IU} / l$ & & A24, B35, 62, Cw3 \\
LAP & $123 \mathrm{IU} / l$ & & DPB1*0201, 0501 \\
ChE & $82 \mathrm{IU} / l$ & & DQB1*0401, 0402 \\
ZTT & $18 \mathrm{U} / l$ & & \\
TTT & $15 \mathrm{U} / l$ & & \\
T.chol & $137 \mathrm{mg} / \mathrm{dl}$ & & \\
TG & $134 \mathrm{mg} / \mathrm{dl}$ & & \\
\hline
\end{tabular}

were undetectable in the sera by polymerase chain reaction and reverse transcriptase polymerase chain reaction. Serology for Epstein-Barr virus (EBV), cytomegalovirus (CMV), herpes simplex virus (HSV) and human herpes virus-6 (HHV-6) was also negative. The anti-nuclear antibody (ANA) titer was 1:160 with a diffuse pattern. Antismooth muscle antibody (ASMA), anti-mitochondrial antibody (AMA), and perinuclear anti-neutrophilic cytoplasmic antibody (pANCA) were negative in the sera. The serum immunoglobulin $G$ level was elevated to $2,110 \mathrm{mg} / \mathrm{dl}$. Haplotyping shows that the patient belonged to HLA-DR4. The lymphocyte stimulation test using minocycline was negative. Liver atrophy, hepatosplenomegaly, and ascites were absent by abdominal ultrasonography and computed tomography. Neither esophageal nor gastric varices were detected by upper gastrointestinal endoscopy.

A liver biopsy revealed the presence of collapsed hepatocytes, mainly in acinar zone 3 . Ballooned hepatocytes and acidiphilic degradation was shown throughout the acini. Kupffer cells were enlarged and formed clumps mainly in the parenchyma, but also in the portal area. In addition, there was infiltration of the acini by inflammatory cells, including lymphocytes and plasma cells. Some portal tracts were infiltrated with lymphocytes and plasma cells, however, fibrosis and scar formation were not apparent (Fig. 1A, B).

A probable diagnosis of drug-induced hepatitis was considered and minocycline therapy was discontinued, although the other possible diagnoses, including minocycline-induced AIH or spontaneous AIH, were not completely discarded. Because of progressive deterioration in liver function, steroid therapy with a daily dose of $1 \mathrm{~g}$ of methyl prednisolone was started. The levels of serum bilirubin and transaminase decreased dramatically and approached the normal limits. The serum immunoglobulin G level normalized, and the titer of ANA fell to 1:40.

Laparoscopy and a second liver biopsy were performed 10 weeks after discontinuation of minocycline. Laparoscopy demonstrated small depressions on the liver surface; however, neither reddish markings nor grooved-like and coarse depressions were present. Liver size was normal and splenomegaly was not detected. Second biopsy specimens of the liver revealed moderate condensations of reticulin and small 


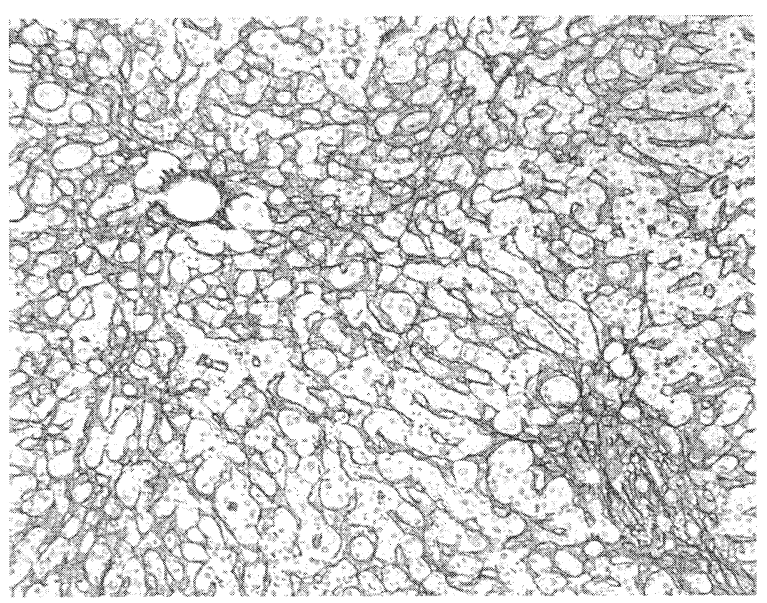

A

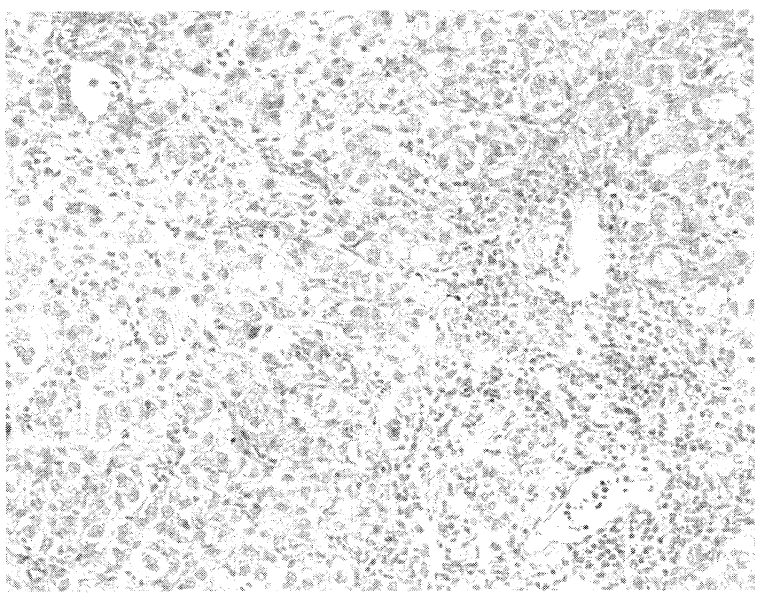

$\mathrm{B}$

Figure 1. Liver biopsy specimen on admission. A: Collapse of hepatocytes was observed mainly in acinar zone 3 (silver stain, $\times 150$ ). B: Ballooned liver cells and acidiphilic degradation were present throughout the acini. In the portal tract, inflammatory cell infiltrates, predominantly lymphocytes and plasma cells, were observed; however, fibrosis and scar formations were not apparent (HE stain, $\times 150)$.

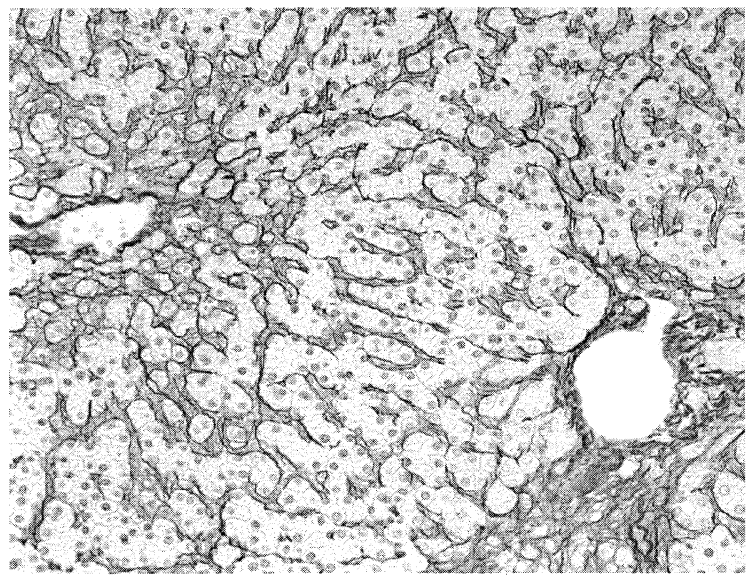

A

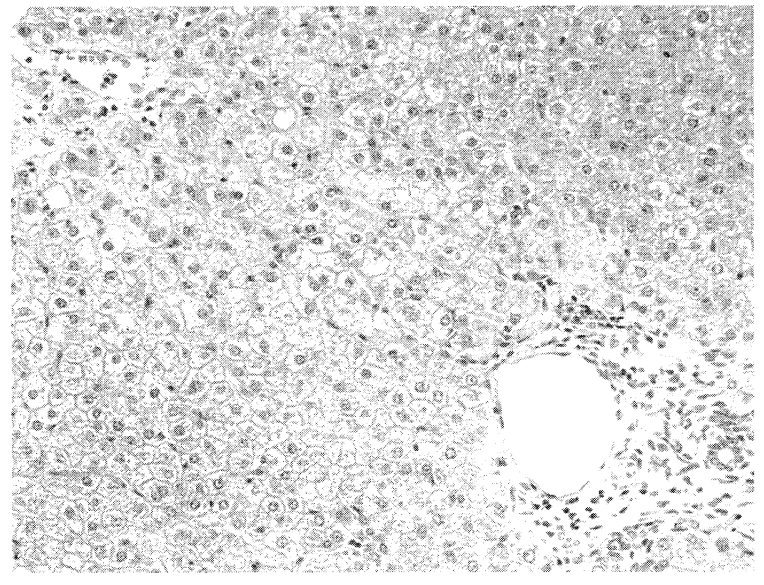

$\mathrm{B}$

Figure 2. Liver biopsy specimen obtained 10 weeks after discontinuation of minocycline. A: Moderate condensations of reticulin were apparent in acinar zone 3 (silver stain, $\times 200$ ). B: Liver cell damage was dramatically improved and small regenerative hepatocytes were seen in acinar zone 3 (HE stain, $\times 200$ ).

regenerative hepatocytes in acinar zone 3 . In addition, dramatic reductions in inflammatory cell infiltrates and damage to hepatocytes were seen compared to those of the first biopsy specimens (Fig. 2A, B). Clumps of Kupffer cells were present in the parenchyma and portal area. Portal tract infiltrations and fibrosis were few.

The patient was treated oral corticosteroids for 3 months. Her liver function tests remained normal after corticosteroid discontinuation.

\section{Discussion}

The patient described here had no risk factors for liver disease, and viral markers were negative. She had been taking minocycline for a long period for the treatment of acne vulgaris. She developed hepatitis, and the titers of ANA and serum immunoglobulin $\mathrm{G}$ were elevated. After discontinuation of minocycline and initiation of corticosteroid therapy, she improved completely, both biochemically and clinically. 
According to WHO documentation, a total of 8,025 persons showed reactions to minocycline, and $493(6 \%)$ of these reactions involved the liver (14). Minocycline hepatotoxity was first presented as microvesicular steatohepatitis in patients treated intravenously with high doses of this drug (15). However, these reactions have been reduced drastically and are extremely rare now. Minocycline-induced hepatotoxic reactions have been categorized into two groups. The first group is, those that appear to be attributable to a hypersensitivity reaction with fever, skin rash, arthralgia, and hypereosinophilia. All reported cases of hypersensitivity reactions occurred within 35 days of the start of minocycline therapy (14).

The second group is, those that can be classified as AIH. These cases are also diagnosed as minocycline-induced hepatitis with autoimmune features (13). This reaction is presented as an autoantibody-positive in sera without eosinophilia and other allergic features, and mainly affects young women. A systematic review of the published literature (14) found that $69 \%$ of patients with minocycline-induced AIH were female with a mean age of 22 years. Moreover, AIH developed with a prolonged course of minocycline therapy, usually after a year or more, and a positive titer for antinuclear antibodies was recorded in $90 \%$ of the patients. Thus, our case has features similar to the previously reported cases of minocycline-induced AIH.

Some authors have reported the histologic features of minocycline-induced AIH (8-13). Goldstein et al (12) compared the morphologic features of liver biopsy specimens of minocycline-induced AIH and spontaneous AIH and reported that they are usually identical. The present patient showed histologic features of acute hepatitis; however, patients with spontaneous AIH with histologic features of acute hepatitis have been reported $(16,17)$.

Thus, the serologic and morphologic features of minocycline-induced AIH are very similar to those of sporadic AIH. However, the two conditions differ in outcome. Most of the patients with minocycline-induced AIH recover upon withdrawal of the antibiotics. Eight of 29 reported patients were treated with corticosteroids (5). In our experience, resolution without recurrence after withdrawal of the drug and a short course of corticosteroids favors druginduced AIH rather than spontaneous AIH. We diagnosed our patient's condition as drug-induced hepatitis with autoimmune features, because liver biopsy specimens showed that the parenchymal changes were predominant in acinar zone 3. However, the possibility of minocycline-induced AIH was not completely ruled out, due to the presence of inflammatory cell infiltrates including plasma cells in portal area at the time of the first biopsy.

The mechanism by which minocycline induces hepatitis is unknown. Herzog et al (11) analyzed the serum of an affected patient and found multiple autoantibodies, including those to 50- and 90-kd proteins on human hepatoma cell lines. They speculated that an antibody reaction against a yet unknown metabolite of minocycline cross-reacts with several microsomal cytochromes. Unique HLA types of patients with minocycline-induced AIH have not been investigated yet.

Reported cases of minocycline-induced $\mathrm{AIH}$ or minomycine-induced hepatitis with autoimmune features, have been mainly from Europe and the United States (4-6, 8-13). However, follow-up biopsies after steroid therapy in the same patients have not heretofore been reported in detail and their characteristics have not been clarified. Few case reports in the literature originate from Japan. The reason for this is unknown; however, it may depend on differences in genetic background or popularity of the drug.

However, the possibility of lack of awareness of this condition was not completely discarded. Clinical suspicion and early recognition of possible drug causes of hepatitis are critically important, since withdrawal of the causative drug leads to a rapid recovery. Minocycline is a widely used antibiotic and is sometimes administered for prolonged periods. In order to prevent serious complications, the possibility of drug-induced hepatitis should be considered when liver dysfunction or systemic symptoms develop during minocycline therapy.

\section{References}

1) Rossman RE. Minocycline treatment of tetracycline resistant and tetracycline-responsive acne vulgaris. Cutis 27: 196-207, 1981.

2) Cohen PM. A general practice study investigating the effect of Minocin $50 \mathrm{mg}$ b.d. for 12 weeks in the treatment of acne vulgaris. J Int Med Res 13: 214-221, 1985.

3) Goulden V, Glass D, Cunliffe WJ. Safety of long-term high-dose minocycline in the treatment of acne. Br J Dermatol 134: 693-695, 1996.

4) Elkayam O, Yaron M, Caspi D. Minocycline-induced autoimmune syndromes: an overview. Semin Arthritis Rheum 28: 392-397, 1999.

5) Eichenfield AH. Minocycline and autoimmunity. Curr Opin Pediatr 11: 447-456, 1999.

6) Knowles SR, Shapiro L, Shear NH. Serious adverse reactions induced by minocycline: report of 13 patients and review of the literature. Arch Dermatol 132: 934-939, 1996.

7) Masson C, Chevallier A, Pascaretti C, Legrand E, Bregeon C, Audran M. Minocycline related lupus. J Rheumatol 23: 2160-2161, 1996.

8) Gough A, Chapman S, Wagstaff K, Emery P, Elias E. Minocycline induced autoimmune hepatitis and systemic lupus erythematosus-like syndrome. Br Med J 312: 169-172, 1996.

9) Teitelbaum JE, Perez-Atayde AR, Cohen M, Bousvaros AB, Jonas MM. Minocycline-related autoimmune hepatitis: case series and literature review. Arch Pediatr Adolesc Med 152: 1132-1136, 1998.

10) Malcolm A, Heap TR, Eckstein RP, Lunzer MR. Minocycline-induced liver injury. Am J Gastroenterol 91: 1641-1643, 1996.

11) Herzog D, Hajoui O, Russo P, Alvarez F. Study of immune reactivity of minocycline-induced chronic active hepatitis. Dig Dis Sci 42: 11001103, 1997.

12) Goldstein NS, Bayati NB, Silverman AL, Gordon SC. Minocycline as a cause of drug-induced autoimmune hepatitis: report of four cases and comparison with autoimmune hepatitis. Am J Clin Pathol 114: 591598, 2000.

13) Bhat G, Jordan J Jr, Sokalski S, Bajaj V, Marshall R, Berkelhammer C. Minocycline-induced hepatitis with autoimmune features and neutrophilia. J Clin Gastroenterol 27: 74-75, 1998.

14) Lawrenson RA, Seaman HE, Sundstrom A, Williams TJ, Farmer RDT. Liver damage associated with minocycline use in acne: a systemic 
ABE et al

reviews of the published literature and pharmacovigilance data. Drug Saf 23: 333-349, 2000.

15) Burette A, Finet C, Prigogine T, DeRoy G, Deltenre M. Acute liver injury associated with oral minocycline. Arch Intern Med 144: 14911492, 1984.

16) Abe M, Hiasa $Y$, Masumoto $T$, et al. Clinical characteristics of auto- immune hepatitis with histological features of acute hepatitis. Hepatol Res 21: 213-219, 2001.

17) Lefkowitch JH, Apfelbaum TF, Weinberg L, Forester G. Acute liver biopsy lesions in early autoimmune ("lupoid") hepatitis. Liver 4: 379386, 1984. 